\title{
Cardiac indicator CK-MB might be a predictive marker for severity and organ failure development of acute pancreatitis
}

\author{
Bing Zhao ${ }^{1 \#}$, Silei Sun ${ }^{1 \#}$, Yihui Wang ${ }^{1}$, Huihui Zhu ${ }^{2}$, Tongtian $\mathrm{Ni}^{1}$, Xing $\mathrm{Qi}^{1}$, Lili Xu ${ }^{1}$, Yuming Wang ${ }^{1}$, \\ Yi Yao ${ }^{1}$, Li Ma ${ }^{1}$, Ying Chen ${ }^{1}$, Jun Huang ${ }^{3}$, Weijun Zhou ${ }^{1}$, Zhitao Yang ${ }^{1}$, Huiqiu Sheng ${ }^{1}$, Hongping Qu ${ }^{4}$, \\ Erzhen Chen ${ }^{1}$, Jian $\mathrm{Li}^{5}$, Enqiang Mao ${ }^{1}$
}

${ }^{1}$ Department of Emergency, Ruijin Hospital, Shanghai Jiao Tong University School of Medicine, Shanghai, China; ${ }^{2}$ Department of Surgical Intensive Care Unit, Shanghai Tongji Hospital, Shanghai, China; ${ }^{3}$ Shanghai Key Laboratory of Hypertension, Department of Hypertension, Ruijin Hospital and Shanghai Institute of Hypertension, Shanghai JiaoTong University School of Medicine, Shanghai, China; ${ }^{4}$ Department of Intensive Care Unit, Ruijin Hospital Affiliated to Shanghai Jiao Tong University School of Medicine, Shanghai, China; ${ }^{5}$ Clinical Research Center, Ruijin Hospital Affiliated to Shanghai Jiao Tong University School of Medicine, Shanghai, China

Contributions: (I) Conception and design: B Zhao, E Mao; (II) Administrative support: L Ma, Y Chen, J Huang, W Zhou, Z Yang, H Sheng, H Qu, E Chen, E Mao; (III) Provision of study materials or patients: None; (IV) Collection and assembly of data: S Sun, Y Wang, H Zhu, T Ni, X Qi, L Xu, Y Wang, Y Yao; (V) Data analysis and interpretation: J Li; (VI) Manuscript writing: All authors; (VII) Final approval of manuscript: All authors.

"These authors contributed equally to the work.

Correspondence to: Enqiang Mao. Department of Emergency, Ruijin Hospital, Shanghai Jiao Tong University School of Medicine, 197 Ruijin Er Road, Huangpu District, Shanghai 200025, China. Email: maoeq@yeah.net; Jian Li. Clinical Research Center, Ruijin Hospital Affiliated to Shanghai Jiao Tong University School of Medicine, Shanghai 200025, China. Email: 1j12116@rjh.com.cn.

Background: The prediction of severe acute pancreatitis (SAP) is the key to providing timely and targeted intensive care for acute pancreatitis (AP). The heart is one of multiple organs involved in the early stage of SAP, but the predictive ability of cardiac dysfunction for SAP remains elusive. We sought to determine if the serum levels of three cardiac indicators (CI) including N-terminal pro-brain natriuretic peptide (NTproBNP), cardiac troponin I (cTNI), and creatine kinase myocardial band (CK-MB) at admission could predict the occurrence of SAP and the development of related organ failure $(\mathrm{OF})$.

Methods: A retrospective, single-center cohort study was conducted on the files of patients presenting to the emergency intensive care unit and medical ward of a regional hospital in Shanghai. Patients diagnosed as having AP and who met the 2012 Atlanta guideline were admitted within 48 hours after disease onset.

Results: Of the $670 \mathrm{AP}$ patients screened, 238 were enrolled into the study and divided into mild acute pancreatitis (MAP) ( $\mathrm{n}=59)$, moderate severe acute pancreatitis (MSAP) ( $\mathrm{n}=123)$, and SAP ( $\mathrm{n}=56)$ groups. No significant difference was found in baseline age, gender, duration from disease onset to admission, comorbidity, or substance abuse. As the levels of three CIs were significantly higher in the SAP group than in the MAP and MSAP groups, the enrolled patients were regrouped into non-SAP and SAP groups for predictive evaluation. Multivariate analysis and nomogram modelling showed that CK-MB, but not cTNI or NT-proBNP predicted the occurrence of SAP [area under curve (AUC) $=0.805$, confidence interval (CI): 0.794-0.905]. Specifically, 89 patients with OF (Modified Marshall score $\geq 2$ ) upon admission were selected and $\mathrm{CK}-\mathrm{MB}$ was shown to predict (AUC $=0.805$, CI: 0.794-0.905) persistent OF ( $\mathrm{n}=48$, duration of OF $>48$ hours) compared to transient organ failure (TOF) ( $n=41$, duration of $\mathrm{OF}<48$ hours).

Conclusions: CIs including NT-proBNP, cTNI, and CK-MB were elevated in the early stage of AP. CK$\mathrm{MB}$ might be used as an efficient predictive biomarker for SAP occurrence and OF development at admission.

Keywords: Severe acute pancreatitis (SAP); severity; biomarker; prediction; cardiac indicator; creatine kinase-MB

Submitted Apr 03, 2020. Accepted for publication Oct 23, 2020.

doi: 10.21037/atm-20-3095

View this article at: http://dx.doi.org/10.21037/atm-20-3095 


\section{Introduction}

Severe acute pancreatitis (SAP) accounts for approximately $20 \%$ of acute pancreatitis (AP) cases and has a reported mortality rate as high as $24 \%$ (1). Key measures to improve the outcome of SAP are its early prediction and diagnosis, which allow physicians to provide timely intensive care. According to the 2012 Atlanta guideline (2), SAP is characterized by persistent organ failure (POF) which is defined by a Modified Marshall score (MMS) >2 and the duration of organ failure (OF) lasting $\geq 48$ hours. This definition usually delays the diagnosis of SAP, especially in those patients who are admitted within 48 hours of disease onset. Despite a plethora of predictive tools including scoring systems and laboratory tests, none has been shown to be clearly superior, while the predictive power remains modest (3).

Cardiac dysfunction has been reported in the early stage of AP (4) and is usually demonstrated by echocardiography (cardiac regional wall-motion abnormalities and impaired diastolic function) and electrocardiography (T-wave flattering and ST-segment depression) (5). However, both methods lack specificity, are relatively complex, and require specialist assistance, which may restrain their application in the prediction of SAP. Serum cardiac indicators (CIs) including $\mathrm{N}$-terminal pro-brain natriuretic peptide (NTproBNP), cardiac troponin I/T (cTNI/T), and creatine kinase myocardial band (CK-MB) are widely used and easily obtained in cardiac ischemic disease and cardiac dysfunction. Recently, these biomarkers have been shown to be well correlated with the severity and prognosis of sepsis, a critical illness $(6,7)$.

Like sepsis, the early pathogenesis of SAP is also characterized by the release of inflammatory cytokines and reactive oxygen species (ROS) which exert direct damage on the myocardium (8). Although NT-proBNP, troponin, and $\mathrm{CK}-\mathrm{MB}$ have been shown to be elevated in the early stage of AP (9), the results remain inconsistent and their ability to predict the severity of SAP is still not well defined. In a prospective study $(n=37)$ Pezzilli et al. $(10,11)$ reported that no difference in serum cTNT, CK-MB, or NT-proBNP levels were found between mild and severe AP patients. However, their findings were inconsistent with another study which showed that the serum level of BNP correlated with AP severity (6).

We conducted a retrospective cohort study to determine if the serum levels of CIs, including NT-proBNP, cTNI, and CK-MB within 48 hours of disease onset could predict the occurrence of SAP. Specifically, SAP patients with single or multiple OF (MMS $\geq 2$ ) at admission and with OF that lasted $>48$ hours were defined as POF. Mild severe acute pancreatitis (MSAP) patients who exhibited OF at admission and but recovered from OF within 48 hours were defined as TOF. We further evaluated the predictive ability of CIs on OF development (POF or TOF) at admission. We present this work in accordance with the STROBE guideline checklist (available at http://dx.doi.org/10.21037/ atm-20-3095).

\section{Methods}

\section{Cobort subjects}

The study was approved by the Institutional Ethics Committee of Ruijin Hospital and was conducted in accordance with the Declaration of Helsinki (as revised in 2013). Written informed consent was obtained from each participant or their family members. This single-center, retrospective cohort study was conducted in the emergency department including the intensive care unit and medical ward of the Ruijin hospital which is a regional hospital in Shanghai. The clinical records of 670 AP patients presenting between September 2012 to April 2017 were evaluated (Figure S1). The exclusion criteria for patients were as follows: (I) age $<18$ or $>80$ years; (II) a history of chronic pancreatitis; (III) pregnancy or lactation; (IV) admitted $>48$ hours after the onset of abdominal pain; (V) illnesses known to increase CI (NT-proBNP, cTNI, CK$\mathrm{MB})$ levels, including chronic heart disease, coronary artery disease, congestive cardiac failure, severe valvulopathy and/ or cardiomyopathy, chronic obstructive pulmonary disease, chronic renal failure, cancer, immune deficiency disease, diseases of the central nervous system (such as meningitis, brain abscess, cerebral hemorrhage), and hormonal or immunosuppressive medications within 3 months; (VI) a lack of complete CI level data.

\section{Diagnosis}

According to the 2012 revised Atlanta guideline (2), the diagnosis of AP requires at least two of the following three features: (I) abdominal pain consistent with the disease; (II) serum lipase activity (or amylase activity) at least three times greater than the upper limit of normal levels; or (III) characteristic findings from abdominal imaging.

The MMS system was used to evaluate the respiratory, 
renal, and circulation variables. The type and duration of $\mathrm{OF}$ were also recorded. Mild AP (MAP) was characterized as a lack of either OF or local or systemic complications. MSAP was characterized as transient organ failure ( $<48$ hours) or local or systemic complications, and SAP was characterized as POF (>48 hours). Etiologies including biliary (12), lipidemic (13), and alcoholic (14) pancreatitis were also determined.

\section{Data collection}

General information including age, gender, duration since disease onset to admission, comorbidity (diabetes, hypertension), and substance abuse (tobacco and alcohol) were collected from the electronic medical records. The daily intake of alcohol did not meet the criteria of alcoholic pancreatitis (14). Serum level of CIs (NT-proBNP, cTNI, $\mathrm{CK}-\mathrm{MB}$ ) and inflammatory markers procalcitonin (PCT) and C-reactive protein (CRP) on admission were obtained from the central laboratory of Ruijin Hospital according to standard protocol. Information concerning multiple organ (lung, respiratory, circulatory) failure indicators at admission including the oxygenation index (OI), serum level of creatine, systemic arterial pressure, and mean arterial pressure, along with that concerning the organ support provided (mechanical ventilation, renal replacement therapy, vasoactive agent) was recorded. Several severity scoring systems including the Acute Physiology and Chronic Health Evaluation II (APACHE II), Sequential Organ Failure Assessment (SOFA), Bedside Index for Severity in Acute Pancreatitis (BISAP), and the Modified Computed Tomography Severity Index (MCTSI) were collected upon admission. Outcome indicators including the length of stay (in hospital), surgery, and in-hospital mortality were analyzed.

All patients received intensive management including controlled fluid resuscitation, support of organ function, and enteral nutrition by the same clinical team to reduce potential bias (15).

\section{Statistical analysis}

All statistical tests were conducted using SAS v. 9.2 (SAS Institute Inc., USA) and R project v. 3.5.2 (The R Foundation for Statistical Computing, Vienna, Austria. http://www.r-project.org). Nominal data are reported as frequency with percentage. Quantitative data are reported as mean and standard deviation (SD) or median, lower, and upper quartiles (Q1; Q3), depending on the normality of each variable's distribution (as assessed with Shapiro-Wilk's test). The contingency tables were analyzed with Pearson's chi-squared test. In the case of three groups, the whole contingency table was analyzed first, and then the pairwise comparisons were performed using Pearson's chi-squared test with Bonferroni correction. Due to the non-normal distribution of most quantitative variables, Kruskal-Wallis's analysis of variance (with post-hoc comparisons using Siegel and Castellan method) was applied when the three groups were compared, while Mann-Whitney's test was performed when two groups were compared. The optimal value of cutoff of related indicators was determined using the analysis of time-dependent receiver operating characteristic (ROC) curve. The univariate logistic regression and the backward stepwise (entry and removal probability were 0.05 and 0.10 respectively) multivariate logistic regression models were fitted with SAP and OF being dependent variables respectively, and we calculated the odds ratio (OR) together with corresponding 95\% confidence interval (CI). All analyses in this study were performed with two-sided $\mathrm{P}$ values at a $5 \%$ significance level. The regression modelling strategies (rms) package within $\mathrm{R}$ was applied to construct a nomogram model (Figures S2 and S3) on the basis of independent determinants identified in the multivariate logistic regression. The overall survival (OS) was estimated using the Kaplan-Meier method, and the log-rank test was applied to compare the survival difference between nonSAP and SAP groups. The hazard ratio (HR) with 95\% CI was estimated with a Cox proportional hazards model.

\section{Results}

\section{Characteristic of patients}

As shown in Table 1, 238 patients were enrolled and divided into the MAP $(n=59)$, MSAP $(n=123)$, and SAP $(n=56)$ groups according to the 2012 Atlanta guideline (2). Among the three groups, there was no significant difference in baseline items including age, gender, duration from disease onset to admission, comorbidity including hypertension and diabetes, or substance abuse including tobacco and alcohol. The etiologies of AP including biliary, lipidemic, and alcoholic are also displayed. The oxygenation index and serum creatinine upon admission were significantly lower in the SAP group than in the MAP and MSAP groups, and the ratio of organ supports including mechanical ventilation and renal replacement therapy were also highest in the SAP group. The highest serum levels upon admission of the two 
Table 1 characteristics of AP patients

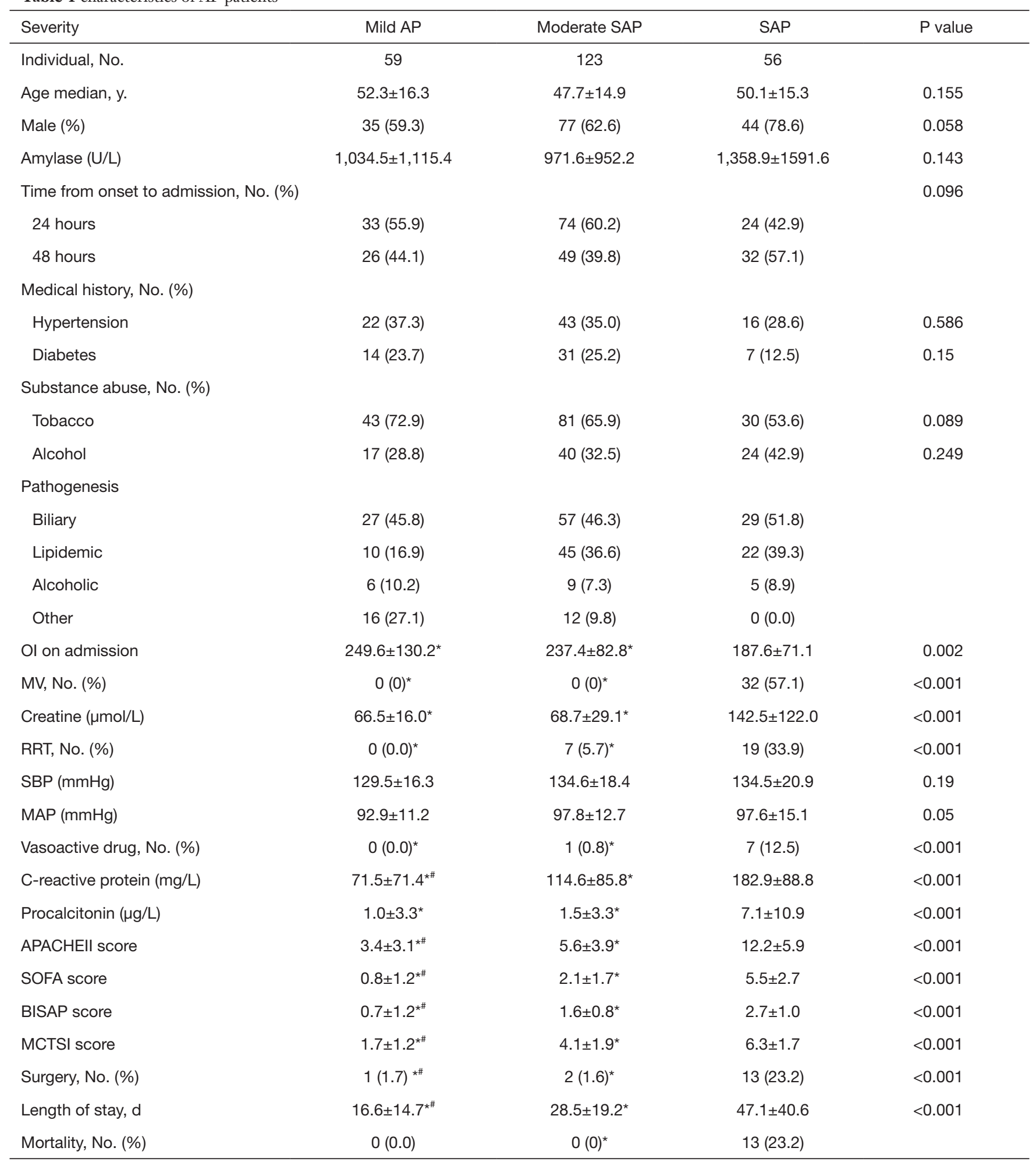

*compared to SAP P<0.05; "compared to MSAP, P<0.05. TG, Triglyceride; OI, oxygenation index; MV, mechanical ventilation; RRT, renal replacement therapy; SBP, systemic blood pressure; MAP, mean arterial pressure; APACHEII, Acute Physiology and Chronic Health Evaluation II; SOFA, Sequential Organ Failure Assessment; BISAP, Bedside Index for Severity in Acute Pancreatitis; MCTSI, Modified Computed Tomography Severity Index; CTSI, CT severity index. 
A

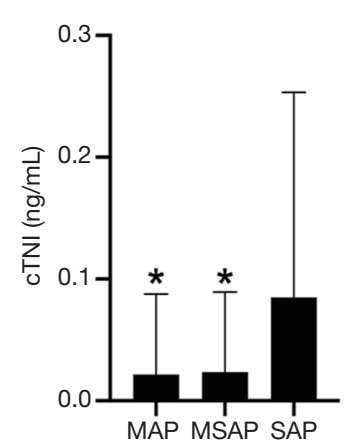

B

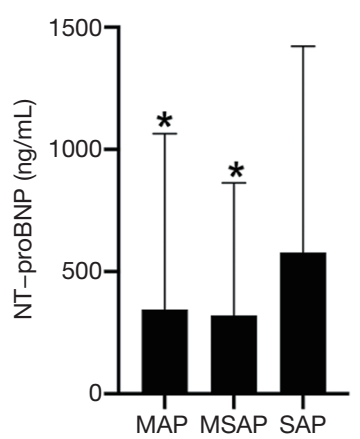

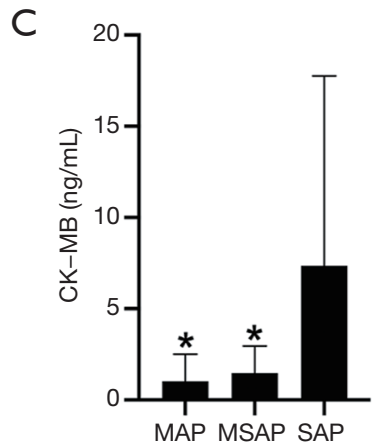

Figure 1 Serum Levels of CIs among MAP, MSAP, and SAP patients. *compared to SAP, $\mathrm{P}<0.05$. CI, cardiac indicators; AP, acute pancreatitis; MAP, mild acute pancreatitis; MSAP, moderate severe acute pancreatitis; SAP, severe acute pancreatitis; NT-proBNP, N-terminal pro-brain natriuretic peptide; cTNI, cardiac troponin I, CK-MB, creatine kinase myocardial band.

inflammatory markers, PCT and CRP, were in the SAP group. Several scoring systems related to severity including the APACHEII, SOFA, BISAP, and MCTSI increased with the severity of dose, and the same trends were also observed in prognosis indicators including the surgery ratio and length of stay. No patients died in the MAP or MSAP groups and mortality in the SAP group was $23.2 \%$. All patients were followed up for 1 week following admission during which time the final diagnosis for severity and etiology were determined.

\section{The level of CIs in the MAP, MSAP, and SAP groups}

Figure 1 shows that the level of NT-proBNP in the MAP $(344.8 \pm 719 \mathrm{pg} / \mathrm{mL})$ and MSAP $(321.1 \pm 542.6 \mathrm{pg} / \mathrm{mL})$ groups are slightly higher than normal levels $(0-300 \mathrm{pg} / \mathrm{mL})$, and are significantly lower than those in the SAP group $(577.6 \pm 843.7 \mathrm{pg} / \mathrm{mL})$. The levels of cTNI in the MAP $(0.022 \pm 0.066 \mathrm{ng} / \mathrm{mL})$ and MSAP $(0.024 \pm 0.066 \mathrm{ng} / \mathrm{mL})$ groups were in the normal range $(0-0.04 \mathrm{ng} / \mathrm{mL})$ and the level of cTNI in the SAP group $(0.085 \pm 0.168 \mathrm{ng} / \mathrm{mL})$ was significantly higher than the normal value. The levels of CK-MB in the MAP $(1.025 \pm 1.481 \mathrm{ng} / \mathrm{mL})$ and MSAP $(1.455 \pm 1.479 \mathrm{ng} / \mathrm{mL})$ groups were in the normal range $(0.3-4 \mathrm{ng} / \mathrm{mL})$, while the level of CK-MB in the SAP group $(7.353 \pm 10.41 \mathrm{ng} / \mathrm{mL})$ was significantly higher than normal. The levels of all three CIs were significantly higher in the SAP group than in the MAP and MSAP groups $(\mathrm{P}<0.05)$.

\section{The predictive power of CI for SAP occurrence}

As the levels of the three CIs upon admission were all shown to be higher in the SAP group than the normal level and in the MAP and MSAP groups, we regrouped the enrolled AP patients into two groups, a non-SAP and a SAP group, to evaluate the predictive power of CIs on the occurrence of SAP. The levels of CIs as well as inflammatory biomarkers (CRP and PCT) and several score systems (APACHE II, SOFA, BISAP, and MCTSI) were significantly higher in the SAP group than in the non-SAP group (Figure S4). Furthermore, a multiple logistic regression model (Table 2) showed that CK-MB, CRP, APACHEII, and SOFA were independent determinants for SAP. Patients with higher levels of CK-MB (OR =1.471, 95\% CI: 1.169-1.850), CRP (OR $=1.008,95 \%$ CI: 1.002-1.014), and higher APACHEII (OR $=1.137,95 \%$ CI: $1.012-1.277)$, and SOFA (OR $=2.075$, 95\% CI: $1.535-2.805)$ scores were more likely to develop SAP. The ROC curve (Figure 2) indicated that CK-MB (AUC $=0.805$, CI: 0.794-0.905) had similar predictive accuracy as APACHEII (AUC $=0.866$, CI: 0.818-0.914) and SOFA (AUC =0.896, CI: 0.855-0.937), but showed significantly higher predictive accuracy than did CRP (AUC $=0.734$, CI: 0.664-0.803, $\mathrm{P}=0.0034$ ). Survival analysis was performed between the SAP and non-SAP groups (Figure S5) and a statistically significant difference was observed $(\mathrm{P}<0.05)$.

\section{Predictive effect of $\mathrm{CI}$ on the development of $\mathrm{OF}$}

One of the key clinical problems related to SAP is predicting the development of organ dysfunction. When AP patients exhibit dysfunction of one or multiple organs (lung, kidney, circulatory) upon admission, it is vital to predict whether this will last beyond 48 hours or not, which is the rationale 
Table 2 Univariate and multivariate analysis of indicators predicted for SAP occurrence

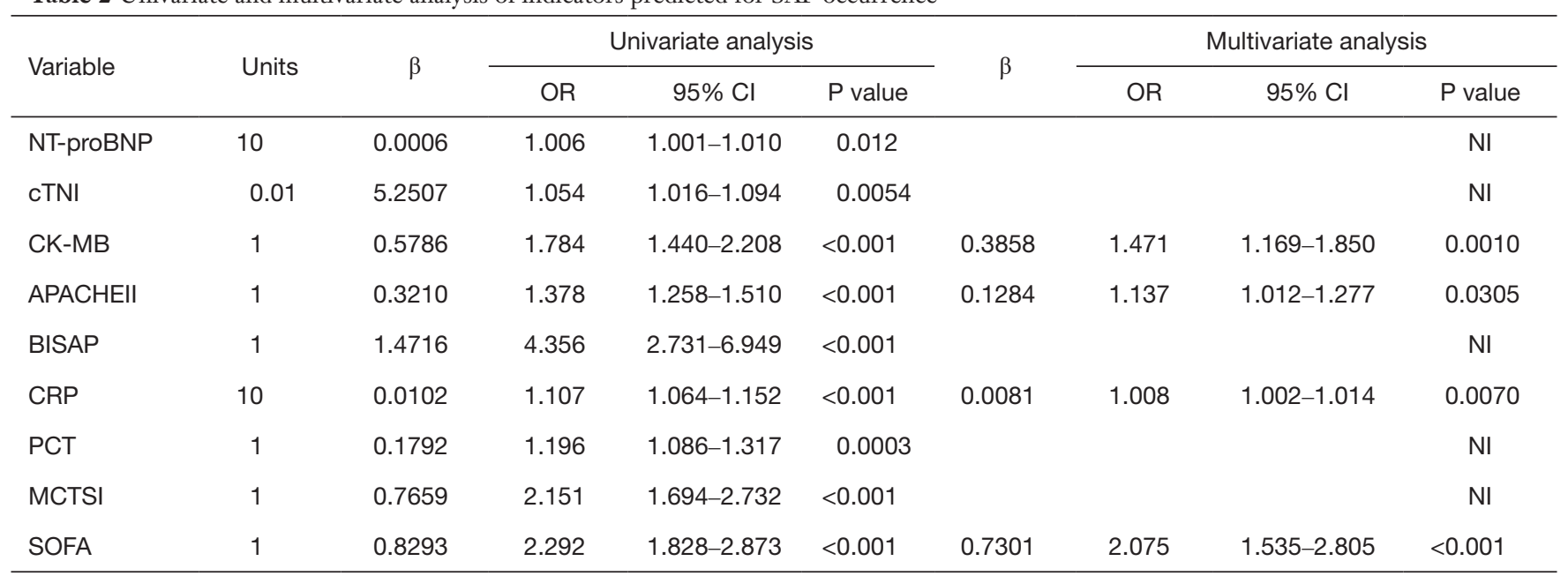

NT-proBNP, N-terminal pro-brain Natriuretic Peptide; cTNI, Cardiac Troponin I, CK-MB, Creatine kinase isoenzymes; APACHEII, acute physiology and chronic health evaluation II; BISAP, acute pancreatitis severity bedside index; CRP, C reactive protein; PCT, procalcitonin; MCTSI, modified computed tomogragh severity index; SOFA, sequential organ failure assessment

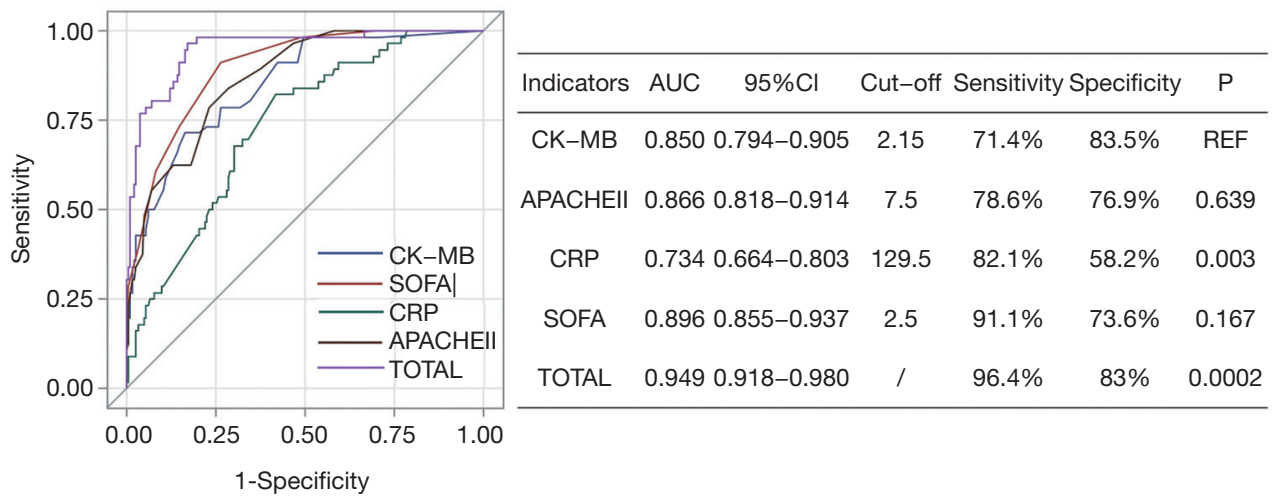

Figure 2 ROC curve of indicators for SAP occurrence. ROC, receiver operating characteristic; CI, cardiac indicators; SAP, severe acute pancreatitis; CK-MB, creatine kinase myocardial band; SOFA, Sequential Organ Failure Assessment; CRP, C reactive protein; APACHEII, Acute Physiology and Chronic Health Evaluation II. Comparison of AUC between CK-MB and other indicators is shown in Table.

behind the definition of MSAP or SAP according to the 2012 Atlanta classification (2). Such prediction not only helps in the early identification of SAP cases with MMS $\geq 2$ upon admission, but also helps in avoiding aggressive treatment and consequent adverse events in MSAP cases with anticipated recovery of OF. With this in mind, 89 patients with dysfunction of one or more organs (MMS $\geq 2$ ) were selected (Table S1) and divided into a TOF group $(n=41$, with transient failure of one or more organs, final diagnosis as MSAP) and a POF group ( $n=48$, with persistent failure of one or more organs, final diagnosis as SAP) according to the duration of OF (beyond 48 hours or not). Compared to the TOF group, the POF group exhibited significantly higher levels of CIs and inflammatory biomarkers (CRP and PCT), along with higher scores on several indices (APACHE II, SOFA, BISAP, and MCTSI) (Figure S6). A multiple logistic regression model (Table 3) showed that CK-MB, BISAP, CRP, and MCTSI were independent determinants for the development of POF. The OF in those patients with higher CK-MB (OR =2.035, 95\% CI: 1.099-3.768), BISAP $(\mathrm{OR}=4.149,95 \%$ CI: 0.964-17.86), CRP $(\mathrm{OR}=1.023$, 95\% CI: $1.008-1.038)$, and MCTSI (OR $=2.234,95 \%$ CI: $1.245-4.008)$ were more likely to progress into POF. The ROC curve (Figure 3) showed that CK-MB (AUC $=0.824$, 
Table 3 Univariate and multivariate analysis of factors predicted for POF

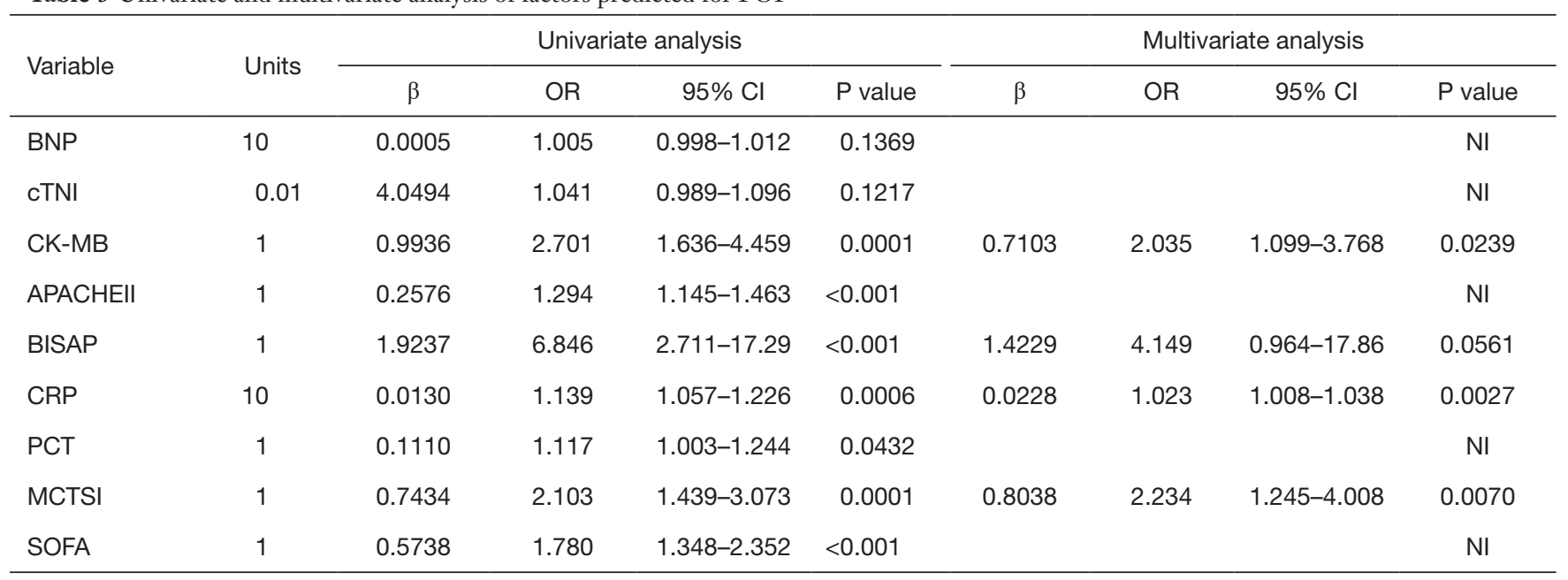

NT-proBNP, N-terminal pro-brain natriuretic peptide; cTNI, cardiac troponin I, CK-MB, creatine kinase-MB; APACHEII, acute physiology and chronic health evaluation II; BISAP, acute pancreatitis severity bedside index; CRP, C-reactive protein; PCT, procalcitonin; MCTSI, modified computed tomography severity index; SOFA, sequential organ failure assessment; POF, persistent organ failure.

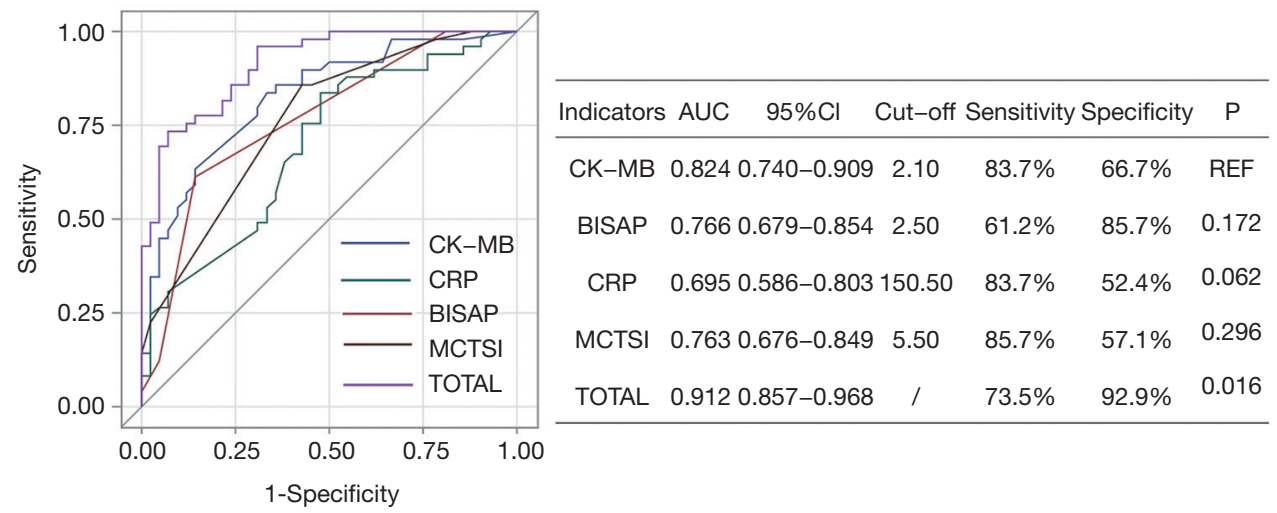

Figure 3 ROC curve of indicators for POF occurrence. Comparison of AUC between CK-MB and other indicators is shown in Table. ROC, receiver operating characteristic; CI, cardiac indicators; POF, persistent organ failure; CK-MB, creatine kinase myocardial band; BISAP, Acute Pancreatitis Severity Bedside Index; CRP, C-reactive protein; MCTSI, Modified Computed Tomography Severity Index; AUC, area under curve.

CI: 0.740-0.909) had similar predictive accuracy as BISAP (AUC $=0.766$, CI: $0.679-0.854)$, CRP $(\mathrm{AUC}=0.695$, CI: $0.586-0.803$ ), and MCTSI (AUC $=0.763$, CI: 0.676-0.849).

\section{Discussion}

We observed a significant differences in the serum levels of three widely used cardiac biomarkers, NT-proBNP, cTNI, and CK-MB, between SAP and non-SAP (MAP and MSAP) patients. The level of CK-MB showed a predictive ability of SAP occurrence while cTNI and pro-BNP did not. Specifically, CK-MB might be used as an independent determinant for POF and for distinguishing a certain group of SAP from MSAP patients who both present with OF (MMS $\geq 2$ ) upon admission.

Several factors have been forwarded as the cause of cardiac injury in the early stage of SAP including the release of large amounts of inflammatory cytokines such as tumor necrosis factor-alpha, interleukin-1 beta, and endotoxin $(16,17)$, in addition to ROS causing cardiomyocyte apoptosis via activating the MAPK pathway or NADPH oxidase hyperactivity (18), or interleukin-1 release (19). 
Cardiac ischemic events caused by profound hypotension, coagulation abnormality, and coronary artery spasm (often induced by electrolyte alteration, such as hypomagnesemia) might play a subordinate role (20). Cardiac injury causes the release of typical cardiac biomarkers such as cTNI, CK$\mathrm{MB}$, and NT-proBNP. Clinical studies also showed elevated cTNI (10), CK-MB (11) and BNP (21) in AP patients, although the results were not consistent. In this study, we showed that the levels of all three CIs were elevated beyond normal ranges within 48 hours after the onset of disease in SAP patients, but not in MAP and MSAP patients, which is partly consistent with the previous studies mentioned above. According to the 2012 Atlanta guideline (2), cardiovascular failure is defined as systolic blood pressure of less than $90 \mathrm{mmHg}$ and no response to fluid therapy according to MMS. However, this definition might not fully reflect cardiac dysfunction and other parameters such as decreased stroke volume and cardiac output (22). Cardiac biomarkers may thus be more useful tools for the direct evaluation of cardiac function.

We chose a cohort of patients that were admitted within 48 hours because of the uncertainty of a diagnosis of SAP upon admission based on the 2012 Atlanta guideline (2) which defines SAP as organ failure lasting for more than 48 hours. This clinical issue needs to be resolved by exploring predictors of the need for intensive care. Multiple logistic regression indicated that an elevated level of CK-MB, but not of cTNI or BNP, could independently predict the occurrence of SAP. Prasada et al. (20) reported elevated serum CK-MB to be associated with AP severity, which is partly consistent with our results, but their enrolled patients were admitted within 7 days after the onset of disease. This might have led to a correlation rather than prediction of SAP as some of their enrolled patients had already been diagnosed with SAP. On the contrary, Barassi et al. (11) demonstrated no difference in CK-MB levels between an SAP group $(n=15)$ and an MAP group $(n=22) 1-3$ days after the onset of disease, although this inconsistency with our results might be due to their relative small cohort size. We also conducted a multivariate logistic analysis and found that CK-MB, CRP, APACHEII, and SOFA could independently predict the occurrence of SAP. Our results are similar to those of a recent study (23) which showed that SOFA had a larger AUC than CRP for the prediction of inhospital mortality.

Aggressive treatment is required as soon as possible when SAP is confirmed but should be avoided for non-SAP patients because of its related adverse events. For patients with $\mathrm{OF}$ of one or more organs upon admission within 48 hours of onset, it is usually difficult to judge if aggressive treatment should be given because it is difficult to ascertain whether OF will last beyond 48 hours (diagnosis of SAP) or not (diagnosis of MSAP). Therefore, there is an urgent need for predictive indicators to assist clinical judgement. The present study showed that CK-MB, as well as BISAP, CRP, and MCTSI could predict a lasting duration ( $>48$ hours) of organ failure at admission. Of these indicators, CK-MB had the largest AUC (0.824) which was significantly higher than that of CRP.

Several prognostic tools including scores and biomarkers have been proposed, including BISAP (24), APACHEII (25), SOFA (3), CRP (22), PCT (26), and MCTSI (27), but their predictive abilities have been inconsistent. A study group in Finland $(28,29)$ attempted to solve this clinical issue by determining the predictive ability of a new biomarker, soluble CD73, but failed.

To the best of our knowledge, very few studies have focused on the predictive effect of CI on AP severity and OF development. In this study, we re-evaluated three CIs representing cardiac failure and found that CK-MB could independently predict SAP occurrence and OF development. This result suggests cardiac injury might be an earlier event that precedes respiratory, renal, and circulatory injuries which are listed in the MMS system for the diagnosis of SAP. Overall, our research emphasizes that the predictive ability of the serum level of CK-MB warrants greater attention in the very early stage of AP.

Some limitations should be noted with this study. First, the sample size was small and the study was conducted in a single-center. In addition, as it is a retrospective study, we did not have data on the CI trend over multiple time points. CK-MB was reported to be mildly elevated on day 1 and normalized in day 3 in a cohort of SAP patients admitted within 7 days after disease onset. This may indicate that the peak level of CK-MB appears in the early stage of SAP and then decreases. In our study, we enrolled a cohort of patients who were admitted within 48 hours after disease onset. It is within this period that CK-MB levels might peak and thus exert their greatest predictive ability.

\section{Conclusions}

Within 48 hours of disease onset, the serum level of CK$\mathrm{MB}$ might be used to predict the occurrence of SAP, more specifically, the development of persistent OF after admission. 


\section{Acknowledgments}

We acknowledge the kind help of Professor Yongting Wang in checking and editing the language of the manuscript. Funding: This work was supported by the Medical Guidance Project of Shanghai Municipal Committee of Science and Technology (No. 18411966400 to Ying Chen), the Shanghai Municipal Commission of Health and Family Planning (No. $2016 Z$ B0206 and ZHYY-ZXYJHZX-1-201702 to Er-Zhen Chen), the Shanghai Municipal Commission of Health and Family Planning (No. 20154 Y0130 to Jun Huang), the National Natural Science Foundation of China (No. 81600501 to Ying Chen and No. 81601665 to Li Ma), and the Medical Guidance Project of Shanghai Municipal Committee of Science and Technology (No. 16411970700 to En-Qiang Mao).

\section{Footnote}

Reporting Checklist: The authors have completed the STROBE guideline checklist, available at http://dx.doi. org/10.21037/atm-20-3095

Data Sharing Statement: Available at http://dx.doi. org/10.21037/atm-20-3095

Peer Review File: Available at http://dx.doi.org/10.21037/ atm-20-3095

Conflicts of Interest: All authors have completed the ICMJE uniform disclosure form (available at http://dx.doi. org/10.21037/atm-20-3095). The authors have no conflicts of interest to declare.

Ethical Statement: The authors are accountable for all aspects of the work in ensuring that questions related to the accuracy or integrity of any part of the work are appropriately investigated and resolved. The study was approved by the Institutional Ethics Committee of Ruijin Hospital and conformed to the provisions of the Declaration of Helsinki (as revised in 2013). Written informed consent was obtained from each participant or their family members. This study was registered in the ClinicalTrials.gov with the registration number of NCT01992198.

Open Access Statement: This is an Open Access article distributed in accordance with the Creative Commons Attribution-NonCommercial-NoDerivs 4.0 International
License (CC BY-NC-ND 4.0), which permits the noncommercial replication and distribution of the article with the strict proviso that no changes or edits are made and the original work is properly cited (including links to both the formal publication through the relevant DOI and the license). See: https://creativecommons.org/licenses/by-nc-nd/4.0/.

\section{References}

1. Beger HG, Rau BM. Severe acute pancreatitis: Clinical course and management. World J Gastroenterol 2007;13:5043-51.

2. Banks PA, Bollen TL, Dervenis C, et al. Classification of acute pancreatitis--2012: revision of the Atlanta classification and definitions by international consensus. Gut 2013;62:102-11.

3. Li M, Xing XK, Lu ZH, et al. Comparison of Scoring Systems in Predicting Severity and Prognosis of Hypertriglyceridemia-Induced Acute Pancreatitis. Dig Dis Sci 2020;65:1206-11.

4. Yegneswaran B, Kostis JB, Pitchumoni CS. Cardiovascular manifestations of acute pancreatitis. J Crit Care 2011;26:225.e11-8.

5. Gyöngyösi M, Takács T, Czakó L, et al. Noninvasive monitoring of hemodynamic changes in acute pancreatitis in rabbits. Dig Dis Sci 1997;42:955-61.

6. Cheng H, Fan WZ, Wang SC, et al. N-terminal probrain natriuretic peptide and cardiac troponin I for the prognostic utility in elderly patients with severe sepsis or septic shock in intensive care unit: A retrospective study. J Crit Care 2015;30:654.e9-14.

7. Sheyin O, Davies O, Duan W, et al. The prognostic significance of troponin elevation in patients with sepsis: a meta-analysis. Heart Lung 2015;44:75-81.

8. Marciniak A, Walczyna B, Rajtar G, et al. Tempol, a Membrane-Permeable Radical Scavenger, Exhibits Anti-Inflammatory and Cardioprotective Effects in the Cerulein-Induced Pancreatitis Rat Model. Oxid Med Cell Longev 2016;2016:4139851.

9. Thandassery RB, Choudhary N, Bahl A, et al. Characterization of Cardiac Dysfunction by Echocardiography in Early Severe Acute Pancreatitis. Pancreas 2017;46:626-30.

10. Pezzilli R, Barassi A, Iammarino MT, et al. Is troponin $T$ a useful marker of myocardial damage in acute pancreatitis? A prospective time course study. Dig Liver Dis 2013;45:347-8.

11. Barassi A, Pezzilli R, Romanelli MC, et al. Serum markers 


\section{Page 10 of 10}

of myocardial damage in acute pancreatitis: a prospective time course study. Pancreas 2015;44:678-80.

12. van Geenen EJ, van der Peet DL, Bhagirath P, et al. Etiology and diagnosis of acute biliary pancreatitis. Nat Rev Gastroenterol Hepatol 2010;7:495-502.

13. Scherer J, Singh VP, Pitchumoni CS, et al. Issues in hypertriglyceridemic pancreatitis: an update. J Clin Gastroenterol 2014;48:195-203.

14. Lee PJ, Papachristou GI. New insights into acute pancreatitis. Nat Rev Gastroenterol Hepatol 2019;16:479-96.

15. Mao E. Intensive management of severe acute pancreatitis. Ann Transl Med 2019;7:687.

16. Jambrik Z, Gyöngyösi M, Hegyi P, et al. Plasma levels of IL-6 correlate with hemodynamic abnormalities in acute pancreatitis in rabbits. Intensive Care Med 2002;28:1810-8.

17. Meyer A, Kubrusly MS, Salemi VM, et al. Severe acute pancreatitis: a possible role of intramyocardial cytokine production. JOP 2014;15:237-42.

18. Wen Y, Liu R, Lin N, et al. NADPH Oxidase Hyperactivity Contributes to Cardiac Dysfunction and Apoptosis in Rats with Severe Experimental Pancreatitis through ROS-Mediated MAPK Signaling Pathway. Oxid Med Cell Longev 2019;2019:4578175.

19. Li L, Sun Z, Xu C, et al. Adenovirus-mediated overexpression of sST2 attenuates cardiac injury in the rat with severe acute pancreatitis. Life Sci 2018;202:167-74.

20. Prasada R, Dhaka N, Bahl A, et al. Prevalence of cardiovascular dysfunction and its association with outcome in patients with acute pancreatitis. Indian J Gastroenterol 2018;37:113-9.

21. Bugdaci MS, Oztekin E, Kara E, et al. Prognostic value of increased $\mathrm{B}$ type natriuretic peptide in cases with acute pancreatitis. Eur J Intern Med 2012;23:e97-e100.

Cite this article as: Zhao B, Sun S, Wang Y, Zhu H, Ni T, Qi X, Xu L, Wang Y, Yao Y, Ma L, Chen Y, Huang J, Zhou W, Yang Z, Sheng H, Qu H, Chen E, Li J, Mao E. Cardiac indicator CK-MB might be a predictive marker for severity and organ failure development of acute pancreatitis. Ann Transl Med 2021;9(5):368. doi: 10.21037/atm-20-3095
Zhao et al. CK-MB as predictive marker for severity and OF of AP

22. Zheng W, Zhang L, Long G, et al. Amalgamation of systemic inflammatory response syndrome score with C-reactive protein level in evaluating acute pancreatitis severity in children. Scand J Gastroenterol 2018;53:755-9.

23. Li J, Bu X, Chen X, et al. Predictive value of long noncoding RNA intersectin 1-2 for occurrence and in-hospital mortality of severe acute pancreatitis. J Clin Lab Anal 2020;34:e23170.

24. Hagjer S, Kumar N. Evaluation of the BISAP scoring system in prognostication of acute pancreatitis - A prospective observational study. Int J Surg 2018;54:76-81.

25. He WH, Zhu Y, Zhu Y, et al. Comparison of multifactor scoring systems and single serum markers for the early prediction of the severity of acute pancreatitis. J Gastroenterol Hepatol 2017;32:1895-901.

26. Kolber W, Kuśnierz-Cabala B, Dumnicka P, et al. Serum Urokinase-Type Plasminogen Activator Receptor Does Not Outperform C-Reactive Protein and Procalcitonin as an Early Marker of Severity of Acute Pancreatitis. J Clin Med 2018;7:305.

27. Gupta P, Rana P, Bellam BL, et al. Site and size of extrapancreatic necrosis are associated with clinical outcomes in patients with acute necrotizing pancreatitis. Pancreatology 2020;20:9-15.

28. Maksimow M, Kyhälä L, Nieminen A, et al. Early prediction of persistent organ failure by soluble CD73 in patients with acute pancreatitis*. Crit Care Med 2014;42:2556-64.

29. Nieminen A, Maksimow M, Mentula P, et al. Circulating cytokines in predicting development of severe acute pancreatitis. Crit Care 2014;18:R104.

(English Language Editor: B. Draper; Quality Control Editor: J. Gray) 


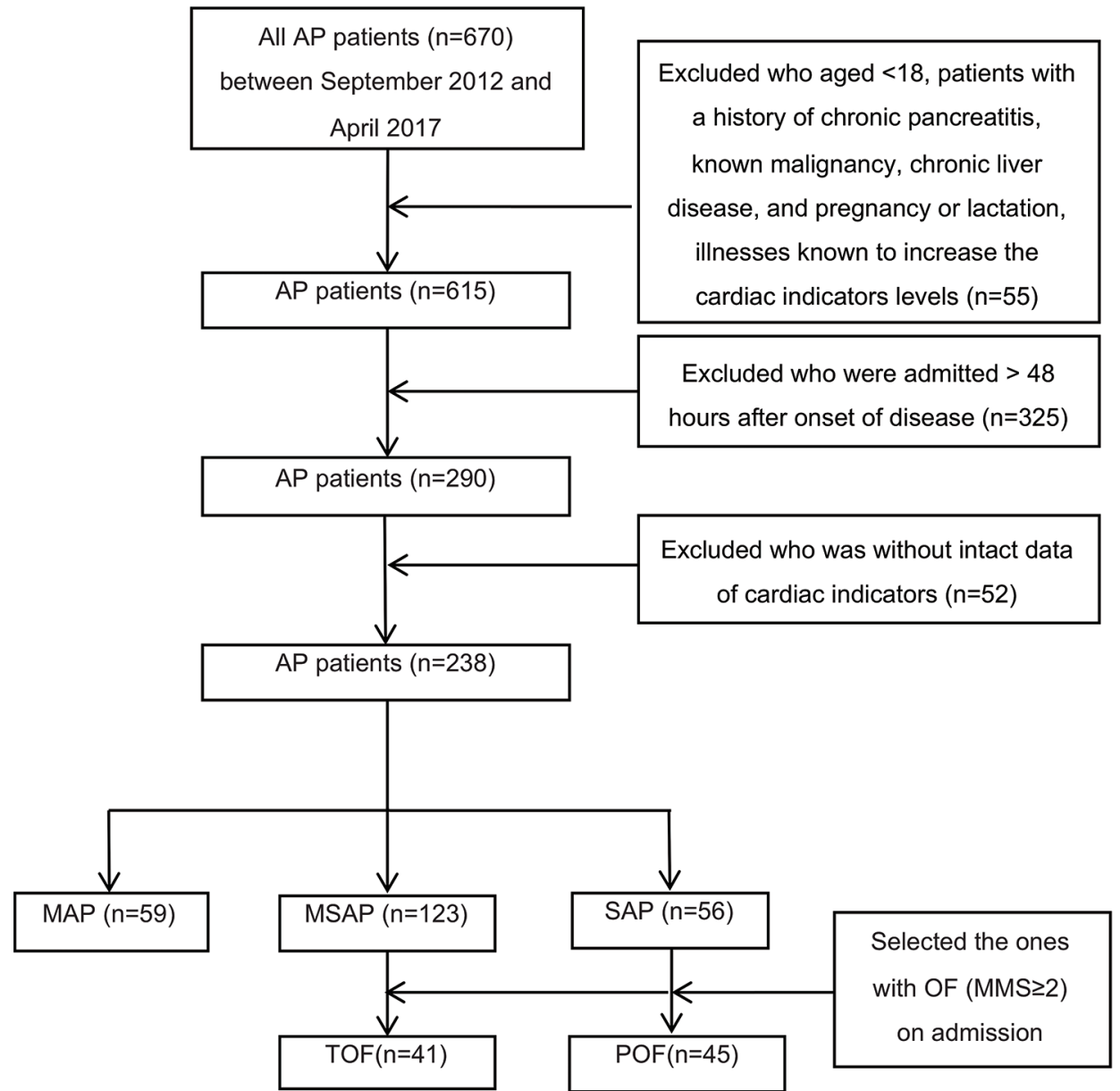

Figure S1 Study flowchart. AP, acute pancreatitis; MAP, mild acute pancreatitis; MSAP, moderate severe acute pancreatitis; SAP, severe acute pancreatitis; OF, organ failure; TOF, transient organ failure; POF, persistent organ failure. 


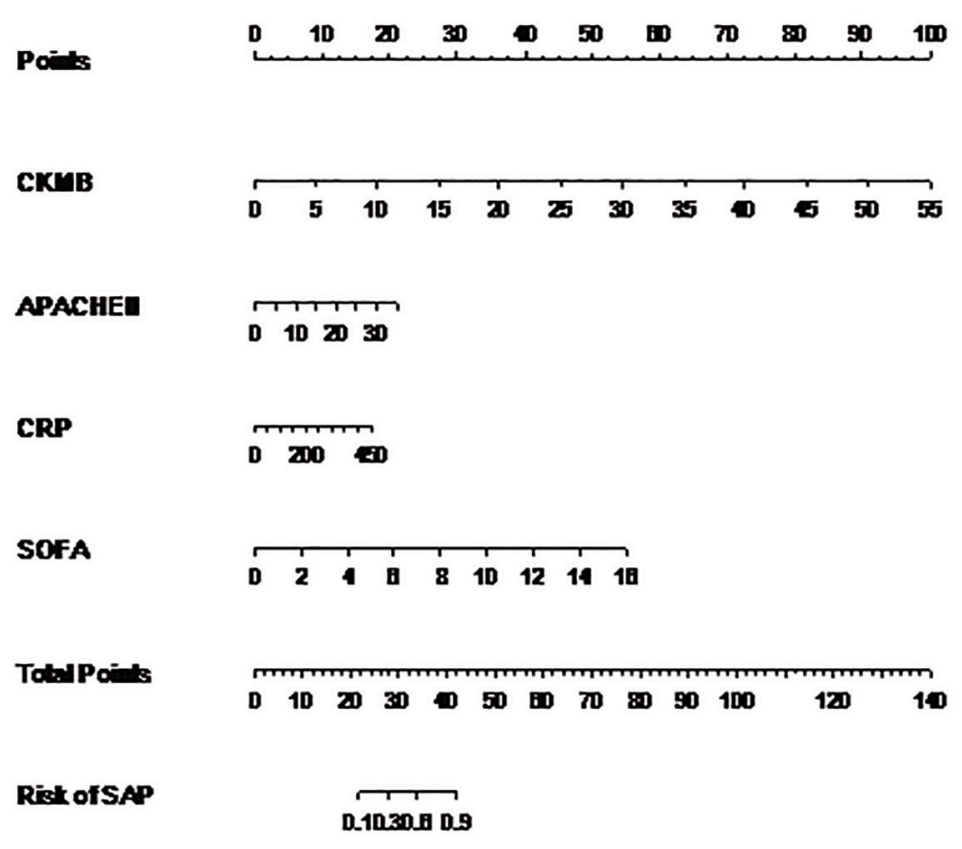

Figure S2 Nomogram for predicting SAP occurrence. CI, cardiac indicators; SAP, severe acute pancreatitis; CK-MB, creatine kinase myocardial band; SOFA, Sequential Organ Failure Assessment; CRP, C-reactive protein; APACHEII, Acute Physiology and Chronic Health Evaluation II.

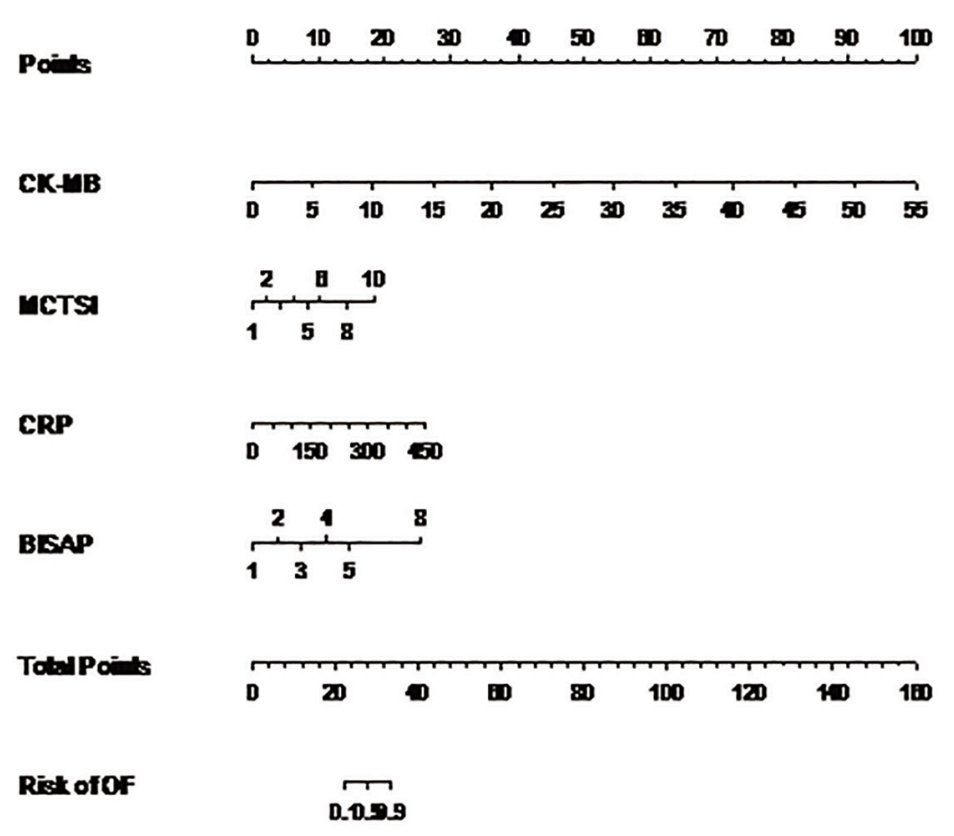

Figure S3 Nomogram for predicting POF occurrence. CI, cardiac indicators; POF, persistent organ failure; CK-MB, creatine kinase myocardial band; BISAP, Acute Pancreatitis Severity Bedside Index; CRP, C-reactive protein; MCTSI, Modified Computed Tomography Severity Index. 

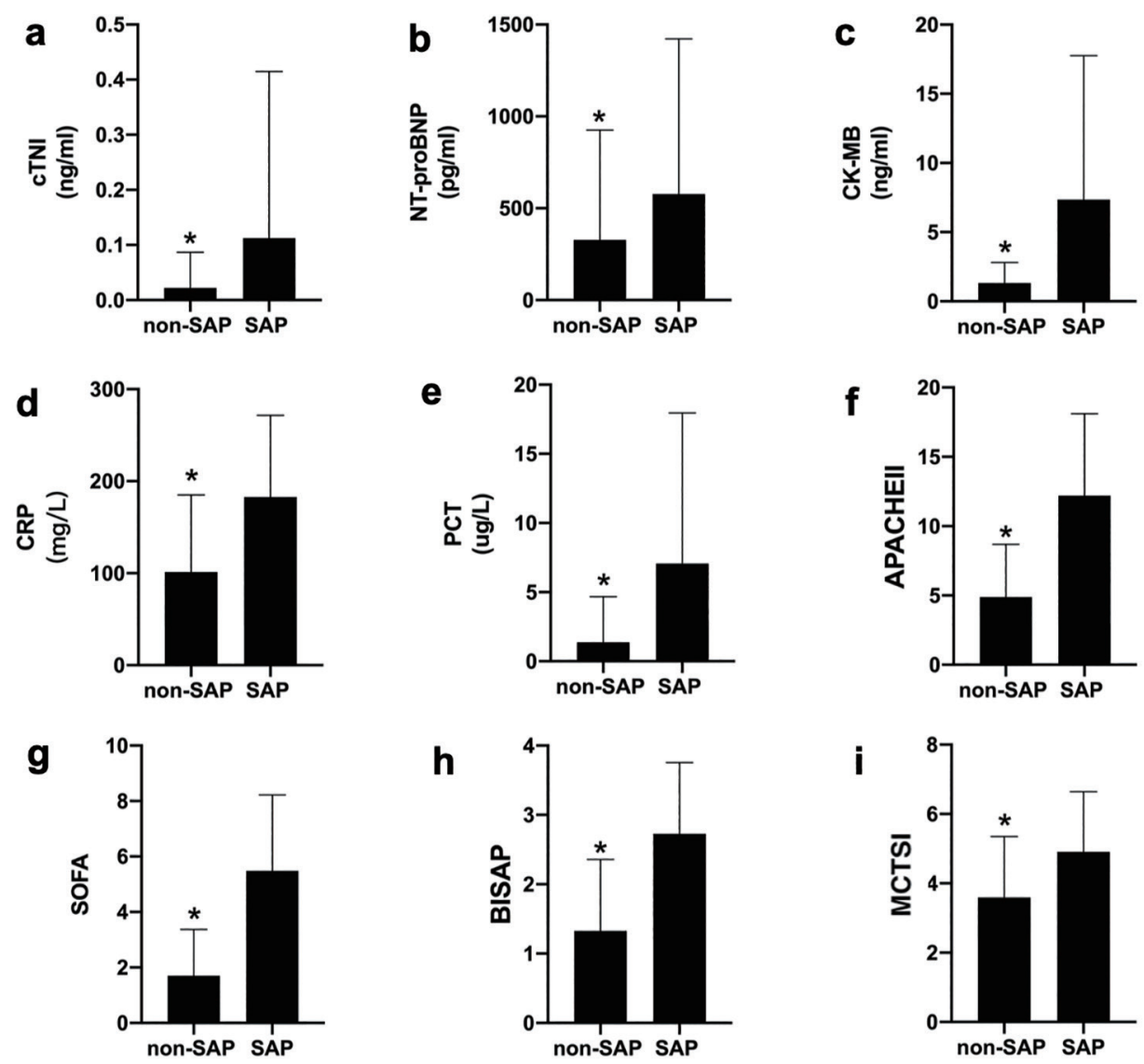

Figure S4 CI, inflammatory biomarkers, and scoring systems between the non-SAP and SAP groups. NT-proBNP, N-terminal pro-brain natriuretic peptide; cTNI, cardiac troponin I, CK-MB, creatine kinase myocardial band; APACHEII, Acute Physiology And Chronic Health Evaluation II; BISAP, Acute Pancreatitis Severity Bedside Index; CRP, C-reactive protein; PCT, procalcitonin; MCTSI, Modified Computed Tomography Severity Index; SOFA, Sequential Organ Failure Assessment; SAP, severe acute pancreatitis. *compared to SAP, P<0.05 


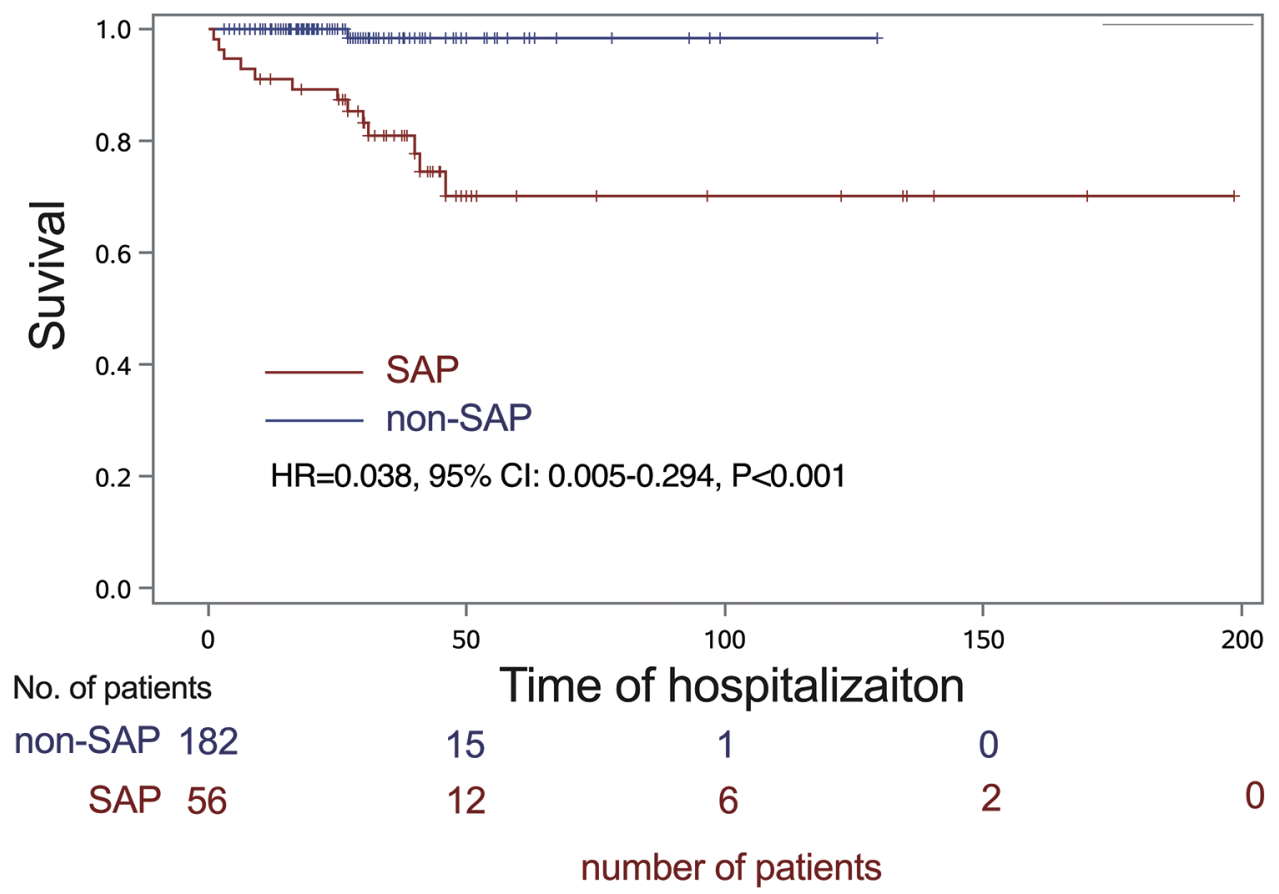

Figure S5 Kaplan-Meier curves for survival analysis of SAP and non-SAP groups. Patients in the non-SAP group enjoyed superior survival compared with those in the SAP group (HR=0.038, 95\% CI: 0.005-0.294, P<0.001). SAP, severe acute pancreatitis; HR, hazard ratio; CI, confidential interval. 

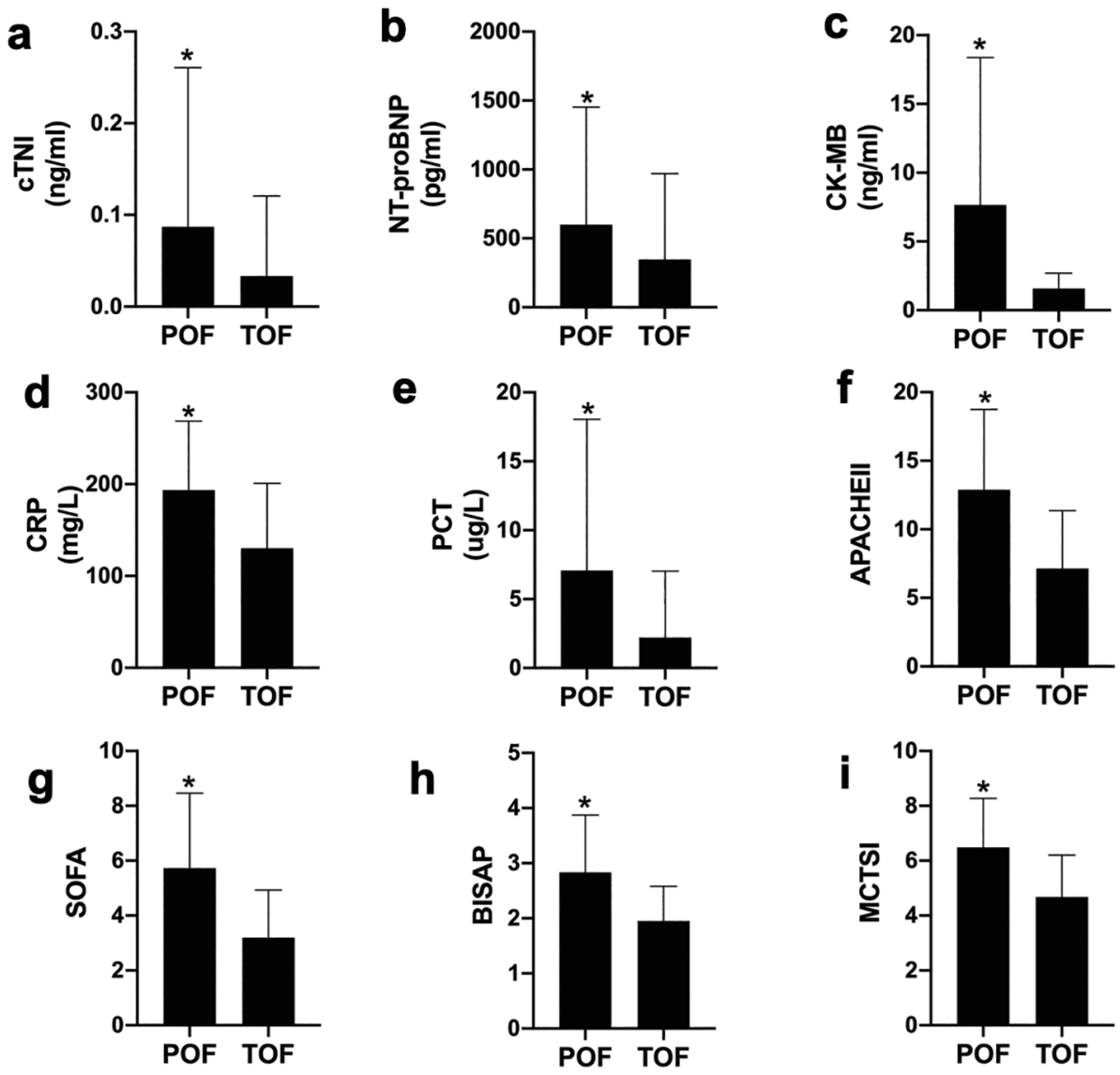

Figure S6 CI, inflammatory biomarkers, and scoring systems between POF and TOF. CI, cardiac indicator; POF, persistent organ failure; TOF, transient organ failure; NT-proBNP, N-terminal pro-brain natriuretic peptide; cTNI, cardiac troponin I, CK-MB, creatine kinase myocardial band; APACHEII, Acute Physiology And Chronic Health Evaluation II; BISAP, Acute Pancreatitis Severity Bedside Index; CRP, C-reactive protein; PCT, procalcitonin; MCTSI, Modified Computed Tomography Severity Index; SOFA, Sequential Organ Failure Assessment. *compared to TOF $\mathrm{P}<0.05$. 
Table S1 Summary of patient with organ failure on admission which then lasted $<48$ or $>48$ hours

\begin{tabular}{ccccc}
\hline & In total & Respiratory & Renal & Cardiovascular \\
\hline OF on admission & 89 & 84 & 30 & 8 \\
OF $<48$ hours & 41 & 39 & 8 & 2 \\
OF $>48$ hours & 48 & 45 & 22 & 6 \\
\hline
\end{tabular}

OF, organ failure. 